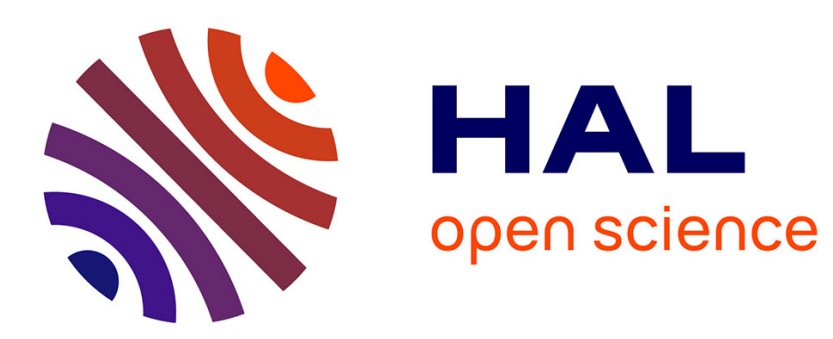

\title{
On the hydrodynamic stability of curved premixed flames
}

Guy Joulin

\section{To cite this version:}

Guy Joulin. On the hydrodynamic stability of curved premixed flames. Journal de Physique, 1989, 50 (9), pp.1069-1082. 10.1051/jphys:019890050090106900 . jpa-00210977

\section{HAL Id: jpa-00210977 https://hal.science/jpa-00210977}

Submitted on 1 Jan 1989

HAL is a multi-disciplinary open access archive for the deposit and dissemination of scientific research documents, whether they are published or not. The documents may come from teaching and research institutions in France or abroad, or from public or private research centers.
L'archive ouverte pluridisciplinaire HAL, est destinée au dépôt et à la diffusion de documents scientifiques de niveau recherche, publiés ou non, émanant des établissements d'enseignement et de recherche français ou étrangers, des laboratoires publics ou privés. 
Classification

Physics Abstracts

$47.20-47.70 \mathrm{~F}$

\title{
On the hydrodynamic stability of curved premixed flames
}

\author{
Guy Joulin \\ Laboratoire d'Energétique et de Détonique, U.A. 193 C.N.R.S., E.N.S.M.A., rue Guil- \\ laume VII, 86034 Poitiers Cedex, France
}

(Reçu le 11 avril 1988, révisé le 21 novembre 1988, accepté le 5 janvier 1989)

\begin{abstract}
Résumé. - On propose une équation non linéaire modèle qui décrit la dynamique de perturbations d'amplitude finie superposées à une flamme faiblement instable et de forme parabolique. Montrant que des solutions admettent une décomposition en pôles, on illustre comment les effets de courbure locaux, la non-linéarité, et l'étirement de la flamme dû à la géométrie rivalisent avec l'instabilité hydrodynamique. Des situations stables, métastables ou conduisant à des structurations latérales sont mises en évidence. Dans le cas de perturbations spatialement périodiques, un analogue non linéaire du critère de Zel'dovich et al. (C.S.T. 24 (1980)) est obtenu. On montre aussi que les dédoublements symétriques et permanents du sommet de la flamme sont non génériques dans la classe de solutions envisagée.
\end{abstract}

\begin{abstract}
We propose a non-linear, model equation describing the dynamics of finite amplitude disturbances superimposed to a two-dimensional, weakly unstable, flame tip of parabolic shape. By showing that solutions of this equation admit a pole decomposition, we illustrate how the local curvature effects, non-linearity and the geometry-induced flame stretch compete with the hydrodynamic instability. Cases of stability, of metastability or leading to « sidecusping » are exhibited. For spatially-periodic disturbances, a non-linear analog to Zel'dovich $e t$ al.'s criterion (C.S.T. 24 (1980)) is obtained. The appearance of steady tip-splitting is also shown to be non-generic in the class of pole-decomposable solutions.
\end{abstract}

\section{Introduction.}

Carefully conducted experiments [1-3] show that curved flames propagating in not too wide tubes may exhibit a smooth steady front, even though they fulfill the two apparently incompatible conditions : (i) their typical transverse dimensions (tube radius, tip radius of curvature) are much larger than the wavelength $\lambda_{c}$ corresponding to the most amplified disturbance of a planar front and (ii) they are locally nearly planar. That such smooth steady curved fronts of large lateral extent also exist for upward propagations [3] clearly shows that gravity is not the mechanism responsible for the flame stability, since body forces are then a destabilizing factor.

A geometry-related mechanism has been proposed by Zel'dovich et al. [4] to explain this anomalous stability on the basis of a WKB-like linear analysis. According to [4] the curved flame is seen as stable despite the Landau-Darrieus $[5,6]$ hydrodynamical instability, for two complementary reasons: (i) because the flow velocity component parallel to the flame 
increases with increasing arc length counted from the tip, the flame shape disturbances of comparatively short wavelengths $\left(\sim \lambda_{c}\right)$ are stretched and are driven to nearly planar ones, which are marginally stable, so that their overall growth is notably reduced ; (ii) again because of the tangential flow, the shape disturbances are advected while they grow, and may impinge the tube walls before having had time to reach a noticeable amplitude. Upon the assumption that thermal noise and $\lambda_{c}$ respectively give the initial amplitude and wavelength of the disturbance, Zel'dovich et al. [4] estimated the transverse flame dimensions (e.g. radius of curvature at its tip) beyond which instabilities may be observed. These arguments and criteria have recently been extended [7] to other curved interfaces (bubbles, Saffman, Taylor finger, etc.) which share this property of anomalous stability with flame fronts ; a recent synthesis and a compilation of related topics can be found in Pelcé [8].

It appeared interesting to us to try extending the above results on flames, at least qualitatively, to the non-linear domain. To this end, we propose a model equation (Sect. 2) derived from the so-called Michelson-Sivashinsky equation [9, 10], after having briefly recalled some properties of the latter. Displaying properties of the model equation in the linear domain (Sect. 3) we further show that it admits a pole-decomposition (Sect. 4), on the basis of which we study the response of a parabolic flame-tip to pulse like disturbances of finite amplitude (Sect. 5). We then study spatially-periodic flame shape disturbances of finiteamplitude (Sect. 6) and we obtain non-linear analogs to the results of reference [4] (Sect. 7) ; the phenomenon of tip-splitting is finally investigated in the class of pole-decomposable solutions (Sect. 8). The paper ends up with a few concluding remarks, open problems and suggestions (Sect. 9).

\section{The M.S. and model equations.}

We found it convenient to use as a starting point the Michelson-Sivashinsky (M.S.) equation $[9,10]$, viz. :

$$
f_{t}+\frac{1}{2} f_{X}^{2}=\mu f_{X X}+I(f, X)
$$

in which the singular integral operator $I(., X)$ is defined as :

$$
I(f, X)=\frac{1}{\pi} \oint_{-\infty}^{+\infty} \frac{f_{X}\left(X^{\prime}, t\right) \mathrm{d} X^{\prime}}{X-X^{\prime}} .
$$

The M.S. equation governs the dynamics of a premixed flame front subject to a weak LandauDarrieus instability $[5,6]$ and has originally been deduced as a leading order result in the limit of weak combustion - induced changes in the mixture density across the flame (hence a weak instability); nonetheless, it has recently been shown to resume the same structure as (2.1) when the asymptotic analysis is made one step further [11]. In (2.1), $t$ is a scaled time, $X$ is a scaled transverse coordinate parallel to the unperturbed flame front $(f \equiv 0)$ and $f(X, t)$ is the flame displacement. The non-local term $I(f, X)$ measures the change in flow velocity just ahead of the flame front. Since the LHS of (2.1) is the small change in absolute flame velocity normally to itself, $\mu f_{X X}$ accounts for the local variations in normal flame velocity relative to the unburned gas, which are due to coupled chemical, diffusive and hydrodynamical phenomena taking place within the flame front thickness. Details on this curvature effect (first introduced phenomenologically by Markstein [12]) and theoretical expressions of the Markstein number $\mu$ can be found in $[13,14]$ and recent experimental values are available in [24] ; it is enough for our present purpose to consider $\mu$ as a given constant characterizing the mixture, and to assume that it is positive. 
Upon linearization about zero, (2.1) admits solutions of the form $\mu \Gamma(k, t) \exp (i k X)$. Since $I(., X)$ corresponds to the multiplication by $|k|$ in Fourier-space, the non-dimensional amplitude $\Gamma(k, t)$ is given in terms of the wavenumber $|k|$ by:

$$
\dot{\Gamma}=\Gamma|k|(1-|k| \mu)
$$

with $\dot{\Gamma}=\mathrm{d} \Gamma / \mathrm{d} t$. One recognizes the destabilizing contribution $|k|$ of the Landau-Darrieus instability, and the cut-off by the stabilizing Markstein effect (provided that $\mu>0$ ) when the wavelength of the disturbance is short enough. We note that two marginal modes are obtained from (2.3), viz. : $k=0$ (translation mode) and $|k|=1 / \mu$ (cellular mode) whereas the wavelength $\lambda_{c}$ of the most amplified disturbance here corresponds to $|k| \mu=1 / 2$.

Upon numerical integration with periodic boundary conditions [10], the M.S. equation often ultimately leads to steadily propagating patterns, viz. : $f(X, t)=-U t+F(X)$, where $U>0$ is the instability-induced increase in flame speed ; $F(X)$ is then a time-independent (but possibly initial-condition-dependent) periodic function consisting of wide parabola-like arcs, the tips of which are convex towards the negative values of $f$ (fresh side), joined by cusplike thin regions (Fig. 1). Let $-U t+F(X)$ be such a «steady solution » to (2.1), or even of its inhomogeneous version obtained by adding a time-independent forcing term $u(X)$ to the RHS (e.g. so as to model a spatial modulation of the incoming fresh flow). Writing $f(X, t)$ as $-U t+F(X)+\phi(X, t)$, one readily obtains :

$$
\phi_{t}+\frac{1}{2} \phi_{X}^{2}+F_{X} \phi_{X}=\mu \phi_{X X}+I(\phi, X) .
$$

About the tip of its parabola-like parts, $F(X)$ is well approximated by $S X^{2} / 2$, where $S>0$ (at least for $S X \ll 1$ ). Motivated by the form that $(2.4)$ then takes, we propose the following equation :

$$
\phi_{t}+\frac{1}{2} \phi_{X}^{2}+S X \phi_{X}=\mu \phi_{X X}+I(\phi, X)
$$

as a model to get better insights into the dynamics of finite amplitude wrinkles superimposed about the tip of a curved steady flame of a (nearly-) parabolic shape (Fig. 2).

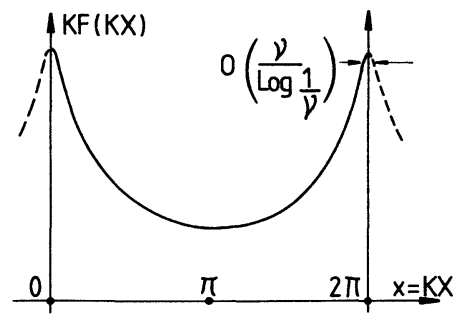

Fig. 1.

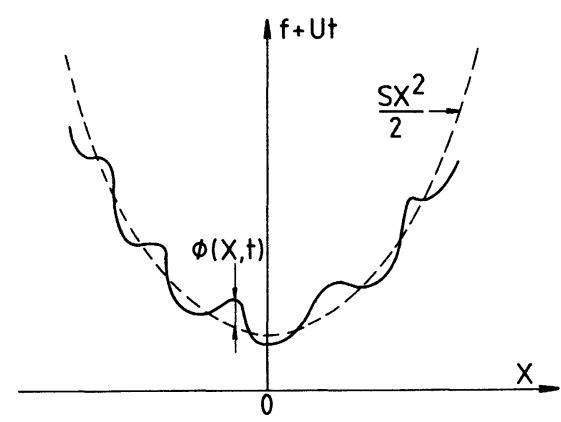

Fig. 2.

Fig. 1. - Shape of a «steady», $2 \pi / K$-periodic solution to (2.1), when $\nu \equiv \mu K$ is small.

Fig. 2. - Definitions of the function $\phi(X, t)$ involved in the model equation (2.5) and of the background parabolic flame shape. 
Due to the very way it has been obtained (2.5) can give valuable information on the anomalous stability of the «steady» solutions - Ut $+F(X)$ to (2.1) itself and on their response to disturbances of finite amplitude, especially when the period $2 \pi / K$ of $F(X)$ is such that $\nu \equiv K \mu$ is small ; even though two parameters $\mu$ and $K$ appear at first glance to control the shape of $F(X)$, it is indeed readily seen, upon an adequate rescaling $(X \rightarrow K X)$ that $\nu=\mu K$ is in fact the only independent parameter ( Markstein length to wavelength ratio). In the limiting case $\nu \rightarrow 0, F(X)$ reads as $g(x) / K$, with $x=K X$; for future reference we note that $g(x)$ is then given by:

$$
g_{x}(x)=-\frac{1}{\pi^{2}} \int_{-\infty}^{+\infty} \cot \left(\frac{x-i y}{2}\right) \log \left(\operatorname{coth} \frac{|y|}{4}\right) \mathrm{d} y .
$$

This limiting form is due to Thual et al. [15] who, using the result of Chen and Lee [16] to exploit the fact that (2.1) admits a pole decomposition (see Sect. 4), obtained «steady » periodic solutions to (2.1) when $\nu \rightarrow 0$. One has also to recall that the experiments of Uberoi [1] did display very nearly parabolic flame shapes and gave a tangential component of the flow velocity which increases linearly with the arclength counted from the tip. Upon use of adequate scalings on $t, X, \phi$, equation (2.5) can thus be viewed as a model to understand the stability of actual flames as well, provided $X$ is interpreted as an arclength; $S$ would then be only proportional to the tip curvature (or to the tangential velocity gradient), the proportionality constant being given by an analysis of the steady front shape and flowfield.

\section{Linear analysis.}

Once linearized for small enough $\phi$ 's, (2.5) admits elementary solutions of the form [25] :

$$
\phi(X, t)=\mu \Gamma(k, t) \exp (i q(t) X) .
$$

Given an initial wave vector $|k|$, the small amplitude $\Gamma(k, t)$ is then determined by:

$$
\begin{gathered}
\dot{\Gamma}=\Gamma q(t)(1-\mu q(t)) \\
\dot{q}=-S q, \quad q(0)=|k|
\end{gathered}
$$

( $\dot{q} \equiv \mathrm{d} q / \mathrm{d} t)$. The amplitude thus follows an equation similar to (2.3), but it involves an instantaneous wavelength $2 \pi / q=2 \pi \mathrm{e}^{S t} /|k|$ which is stretched by the «tangential velocity " component $S X$. Results similar to those of references [4,8] are recovered from equations (3.2), (3.3) by noting first that $\Gamma(k, t \rightarrow \infty)$ saturates to a finite value, since $q(t)$ is driven exponentially rapidly to the marginal state $q=0$ :

$$
\Gamma(k, \infty)=\Gamma(k, 0) \exp \left(\frac{|k|}{S}\left(1-\frac{\mu|k|}{2}\right)\right)
$$

$\Gamma(k, \infty)$ is maximum when $\mu|k|=1$, i.e. when $\phi(0, X)$ is the non-trivial marginal mode corresponding to (2.3). In accordance with [4], there exists a critical value of the tip curvature $S$, for a given initial amplitude $\Gamma(k, 0)$, such that the curved flame is stable $(\Gamma(k, t)$ remains small when $\Gamma(k, 0)$ is) if $S>S_{\mathrm{c}}$. The critical value $S_{\mathrm{c}}$ depends on the initial amplitude and is estimated from (3.4), as :

$$
\mu S_{\mathrm{c}}=O\left(\log ^{-1}(1 / \Gamma(k, 0)) \mu|k|\left(1-\frac{\mu|k|}{2}\right)\right)
$$

since the appearance of a noticeable instability roughly corresponds to $\Gamma(k, \infty)=$ 
$O(1)$. According to (3.5), and in accordance with Zel'dovich et al., a weakly curved flame $(S \ll 1)$ can be stable in an almost noise-free situation, even when its typical transverse dimensions $(\sim 1 / S)$ are well larger than the wavelength (here $4 \pi \mu)$ of the most rapidly growing disturbance of a planar front. When interpreted in the context of an inhomogeneous version of (2.1), this result suggests that the " average wavelength » $\left(\sim 1 / S_{\mathrm{c}}\right)$ of $f(X, t)$ could be selected by the amplitude and the wavelength $2 \pi /|k|$ of a forcing term, added in the RHS of (2.1) to simulate the response of an unstable flame to a weak incoming turbulence, since wider cells would presumably be destroyed by the instability. We note, however, that the weak, logarithmic dependence of $S_{\mathrm{c}}$ on $\Gamma(k, \infty)$ could be difficult to appreciate even by numerical experiments; the consequence of its dependence on $\mu|k|$ is more likely to be attainable.

Conversely, for a given tip curvature $S$, there exists a wavenumber-dependent amplitude $\Gamma_{\mathrm{c}}(k)$ required to trigger noticeable, $O(1)$ wrinkles :

$$
\Gamma_{\mathrm{c}}(k)=O\left(\mathrm{e}^{-(|k| / S)(1-|k| \mu / 2)}\right)>O\left(\mathrm{e}^{-1 / 2 S \mu}\right) \equiv \Gamma_{*} .
$$

The most dangerous disturbance of course corresponds to $\mu|k|=1 . \Gamma_{\mathrm{c}}(k)$ could be exceeded as a consequence of a forcing term simulating an incoming turbulence in (2.1). Other unwanted possible candidates for the excitation of shape disturbances with supercritical amplitudes are the truncation and/or rounded off errors in numerical studies of the M.S. equation when it is integrated over an interval which is large compared to $\mu$. Under such circumstances, (2.6) enables one to compute the tip curvature, upon differentiation and calculation of the resulting integral at $x=\pi$. One obtains $g_{x x}(\pi)=1 / \pi$, whence :

$$
S=K / \pi
$$

Assuming that some numerical noise is generated at the most dangerous mode $|k|=$ $1 / \mu,(3.7)$ and (3.6) give :

$$
\Gamma_{*}=O\left(\mathrm{e}^{-\pi / 2 \mu K}\right)
$$

Michelson and Sivashinsky [17] made one of their numerical integrations of (2.1) on an interval $2 \pi / K$ which is forty times as large as the wavelength $(4 \pi \mu)$ corresponding to the most amplified disturbance of a planar flame, i.e. for $\mu K=1 / 80(S \mu=1 / 80 \pi \cong 5 \times$ $\left.10^{-3}\right)$. Then (3.8) gives $\Gamma_{*}=O\left(\mathrm{e}^{-40 \pi}\right)=O\left(10^{-55}\right)$. Even if a very accurate numerical scheme is employed so as to avoid significant truncation errors, one must be careful to prevent the rounded off errors at the mode $|k|=1 / \mu$ from exceeding this very small value of $\Gamma_{*}$. Therefore, the chaotic travelling waves «issuing » from the cell tips which have been obtained in reference [17] when integrating (2.1) for $K=1 / 80 \mu$ may well be numerical noiseinduced patterns. Even if this is true, these conjectures would not mean that such simulations are meaningless, since noiseless experiments do not exist either but, instead, that they should be re-made (and the above conjecture tested) with a better controlled source of noise than the numerical one. A numerical investigation of this kind has very recently been initiated by Denet [26], who found that the criterion (3.8), deduced from (2.5), is indeed quite relevant, even quantitatively. As pointed out by an anonymous referee, this is a bit surprising, given that (3.8) is based upon the value of $\Gamma(1 / \mu, \infty)$ : approximating the steady solution to (2.1) by a parabola $S X^{2} / 2$ requires that the instantaneous wavenumber $q(t)$ of the disturbance remained much larger than that $(K)$ of the solutions to (2.1), whereas $q$ goes exponentially to zero as $t \rightarrow \infty$, by equation (3.3). However, in the limit $K \mu \rightarrow 0$ (hence $S \mu \rightarrow 0$, owing to (3.7)) it is readily seen that the times $\bar{t}$ at which $q(t)=\mu^{-1} \exp (-S t)$ gets of the order of $S$, are such that $\Gamma(1 / \mu, \bar{t})=O(\Gamma(1 / \mu, \infty))$. Accordingly, since $\Gamma *$ is defined only up to within 
an $O(1)$ factor, the above result (3.8) is relevant in the context of a stability analysis of steady solutions to $(2.1)$ : the maximum growth of $\Gamma(1 / \mu, t)$ is almost completely achieved before (2.5) ceases to be valid, at least in the limit $S \mu \rightarrow 0$. Of course if $\Gamma_{*} \ll \Gamma(1 / \mu, 0) \ll 1$, the non-linear effects must come into play at earlier times than $\bar{t}$.

\section{Pole decomposition.}

In addition to giving qualitatively similar results as Zel'dovich et al.'s in the linear domain, the model equation (2.5) has also the pleasant property that it admits a pole decomposition, i.e. solutions in the form :

$$
\phi(X, t)=f(t)-2 \mu \sum_{\alpha=1}^{2 N} \log \left(X-x_{\alpha}(t)\right) .
$$

The complex numbers $x_{\alpha}(t), \alpha=1 \ldots 2 N$, are the locations of the (movable-) poles of $\phi_{X}$ in the complex $X$-plane, which appear in conjugate pairs for $\phi$ to be real. One already knew that the M.S. equation is pole decomposable $[15,16]$. This was due to a conjunction of the following properties.

- The Fourier transform of $\log \left(X-x_{\alpha}\right)$ vanishes for $k<0$ or $k>0$, depending on the sign of the imaginary part $\operatorname{Im}\left(x_{\alpha}\right)$ of $x_{\alpha}$. Therefore, applying $I(., X)$ to this function yields a result which is proportional to the derivative $1 /\left(X-x_{\alpha}\right)$ since, in Fourier space, $I(., X)$ is the multiplication by $|k|$ whereas $\mathrm{d} / \mathrm{d} X$ corresponds to $i k$.

- In (2.1) (hence in (2.5)) the most divergent terms of $1 / 2 \phi_{X}^{2}$ and $\mu \phi_{X X}$ cancel (1), leaving only terms proportional to $1 /\left(X-x_{\alpha}\right)$ which are of the same type as those coming from $\phi_{t}$ and $I(\phi, X)$. Since all terms must cancel for (2.1) (or (2.5)) to be met, equations were found for the $x_{\alpha}$ 's.

That (2.5) also admits a pole decomposition follows from the trivial identity :

$$
X \frac{\mathrm{d}}{\mathrm{d} X} \log \left(X-x_{\alpha}\right)=1+\frac{x_{\alpha}}{X-x_{\alpha}}
$$

and the corresponding evolution equations for the poles read as $(\alpha=1 \ldots 2 N)$ :

$$
\dot{x}_{\alpha}=2 \mu \sum_{\beta \neq \alpha} \frac{1}{x_{\beta}-x_{\alpha}}-i \operatorname{sign}\left(\operatorname{Im}\left(x_{\alpha}\right)\right)+S x_{\alpha} .
$$

Besides the constraint that they must appear as complex conjugate pairs, the poles may be arbitrarily numerous. Their number (a constant of motion) and their initial locations depend on the initial condition $\phi(X, 0)$; when not present at $t=0$, the poles may presumably be created at $t=0^{+}$by a mechanism similar to that evidenced by Bessis and Fournier [18] for the Burgers' equation. The existence of a pole-decomposition, which enables one to study a continuous non-linear system as a discrete $\mathrm{N}$-body problem for movable singularities, is a rather exceptional event : besides the Burgers, M.S. and Benjamin-Ono equations, which are particular cases of Lee and Chen's [16] non-linear models, pole decompositions have only been obtained for the KDV equation [19] and for a fully non-local problem of interface motion [20].

(1) It may appear surprising that the terms $\phi_{X X}$ (a stabilizing one) and $1 / 2 \phi_{X}^{2}$ (without which (2.1) has no bounded solution) compensate each other. Actually $\phi_{X}^{2}$ also brings in a destabilizing effect, which is responsible for the formation of cusps of $\phi$ when the RHS of (2.1) is set equal to zero. 


\section{Two-pole dynamics.}

The first two terms featuring in the RHS of (4.2) have already been interpreted in [15]. The second one comes from the Landau-Darieus instability, via $I(\phi, X)$; it tends to make the poles collide with the real axis, and to produce logarithmic real singularities in $\phi$. The first term comes from the stabilizing influence of $\phi_{X}^{2}$, and tends to repel the poles off their complex conjugate, thereby preventing a logarithmic singularity of $\phi$ to appear on the real line. The only new, innocuous-looking term introduced by the curved tip geometry is the last one $S x_{\alpha}$ which, if alone, would give $\dot{x}_{\alpha}=S x_{\alpha}$. Since $S$ is positive, $S x_{\alpha}$ tends to shift the poles along the real axis at a speed proportional to their real distance to the origin ; by the same token $S x_{\alpha}$ tends to make their mutual distances increase (stretching the disturbances) and competes with the formation of condensations [15]. At the same time, the new term in (4.2) tends to push the poles off the real axis (stabilizing influence of stretch). As an illustration of its overall influence, we consider the situation where only two poles $x_{1}=a+i b(b>0$, without loss of generality) and $x_{2}=a-i b$ exist. Then (4.2) gives :

$$
\begin{aligned}
& \dot{a}=S a \\
& \dot{b}=\frac{\mu}{b}-1+S b
\end{aligned}
$$

the corresponding expression for $\phi_{X}$ being :

$$
\phi_{X}=-4 \mu \frac{X-a(t)}{(X-a(t))^{2}+b^{2}(t)} .
$$

The solution to (5.1), $a=a(0) \exp (S t)$, clearly displays the disturbance drift in the Lagrangian frame of reference defined by $\dot{X}=S X$. About the evolution of $b(t)$, two cases have to be distinguished :

i) $4 \mu S>1$

If the tip curvature and/or the Markstein number $\mu$ are large enough $(\mu S>1 / 4)$, $b(\infty)=\infty$ obtains whatever the value of $b(0)$, since the RHS of (5.2) is positive for any positive $b$. The disturbance is then damped to zero while being shifted to $X= \pm \infty$;

ii) $4 \mu S<1$

The equation :

$$
S b^{2}-b+\mu=0
$$

has now two real positive roots :

$$
b_{ \pm}=\frac{1}{2 S}(1 \pm \sqrt{1-4 \mu S}) \text {. }
$$

If $b(0)>b_{+}$, then $b(\infty)=\infty$ obtains and the disturbance is again ultimately damped to zero, like in the previous case. If $b(0)<b_{+}$, however, $b(t)$ tends to the stable root $b_{-}$and $\phi(X, t)$ tends to a steady profile while being shifted ; however, at any fixed value of $X, \phi_{X}(X, t \rightarrow \infty) \rightarrow 0$. Consistently with the linear analysis of (2.2), a weakly curved tip is meta-stable against pulse-like disturbances.

It would be interesting to define a threshold initial disturbance amplitude, below which the final amplitude vanishes. However, this is not an obvious task, since the flame front disturbance $\phi(X, t)$ diverges logarithmically as $|(X-a) / b| \rightarrow \infty$ (Eq. (5.3)). We therefore suggest a tentative criterion based upon the shape of $\phi_{X}$. Since the latter achieves its extrema 
at $X-a= \pm b$, and the values $\pm 4 \mu / b$ obtain, one may define the threshold amplitude of the pulse-like disturbances as that for which $\left|\phi_{X}\right|$ exceeds $4 \mu / b_{+}$at $|X-a|=O\left(b_{+}\right)$. One has to realize, however, that the pulse-like disturbances which we consider here are rather peculiar: for them, the terms «weak» and «broad» are synonymous. At any rate, a comparison of this result to (3.8) suggests that the disturbance amplitude needed to ultimately trigger a noticeable pattern depends on the disturbance shape.

It is also worth mentioning that the new term $S x_{\alpha}$ tends to push the remote poles to infinity in the imaginary direction quite rapidly; it seems to be even more legitimate than in the $S=0$ case to only consider a finite number of $x_{\alpha}$ 's.

\section{Spatially periodic disturbances.}

Equation (3.1) indicates that a spatially $2 \pi /|k|$-periodic initial pattern generates a periodic solution to (2.5) with $q=|k| \exp (-S t)$ as wavenumber. We now change the variable from $X$ to $Z=X \exp (-S t)$, thereby modifying (2.5) into :

$$
\psi_{t}+\frac{1}{2} \mathrm{e}^{-2 S t} \psi_{Z}^{2}=\mathrm{e}^{-2 S t} \mu \psi_{Z Z}+\mathrm{e}^{-S t} I(\psi, Z)
$$

where $\psi(Z, t)$ stands for $\phi\left(Z \mathrm{e}^{S t}, t\right)$. Besides the solutions obtained from (4.2) (4.3) by changing the independent variable, (2.5) also admits spatially $2 \pi /|k|$-periodic solutions of the following type :

$$
\psi(Z, t)=g(t)-2 \mu \sum_{\alpha=1}^{2 N} \log \left(\sin \left(\frac{|k|}{2}\left(Z-z_{\alpha}(t)\right)\right)\right)
$$

where $z_{1}, \ldots, z_{2 N}$ again correspond to the poles of $\phi_{X}=\psi_{Z} \exp (-S t)$. This result, which is an extension of reference [15], can be obtained directly from (6.1) by employing the functional equation :

$$
f(a) f(b)+1+f(a-b)(f(a)-f(b))=0
$$

fulfilled by $\cot (Z)$ to transform the non-diagonal terms involved in the expression of $\psi_{Z}^{2}$, or from (4.1) by extensive use of the known [21] pole-decomposition of cot $(Z)$. The ODE's giving the pole motions are now :

$$
\dot{z}_{\alpha}=|k| \mu \mathrm{e}^{-2 S t} \sum_{\beta \neq \alpha} \cot \left(\frac{|k|}{2}\left(z_{\beta}-z_{\alpha}\right)\right)-i \mathrm{e}^{-S t} \operatorname{sign}\left(\operatorname{Im}\left(z_{\alpha}\right)\right) .
$$

The number of poles per cell of $2 \pi /|k|$ lateral extent is again arbitrary, as well as their initial locations.

\section{Two poles per cell.}

When only two poles $z_{1}=A+i B(B>0)$ and $z_{2}=A-i B$ are involved in each cell, (6.4) yields :

$$
\begin{aligned}
\dot{A} & =0 \\
\dot{B} & =|k| \mu \mathrm{e}^{-2 S t} \operatorname{coth}(|k| B)-\mathrm{e}^{-S t}
\end{aligned}
$$

the corresponding form of $\phi(X, t)$ being :

$$
\phi(X, t)=h(t)-2 \mu \log \left(1-\frac{\cos \left(|k| \mathrm{e}^{-S t}\left(X-\mathrm{e}^{S t} A\right)\right)}{\cosh |k| B}\right) .
$$


Once a definition of the mean value of $\phi$ is provided, the function $h(t)$ can be computed, by averaging (6.1) over $Z$. For future reference, we note that the peak-to-peak amplitude of $\phi$ is given by :

$$
\Gamma(k, t)=2 \log \operatorname{coth}(|k| B(t) / 2)
$$

where, consistently with (3.1), $2 \mu$ times the peak-to-peak amplitude $\Gamma$ stands for the difference between the maximum and minimum values of $\phi(X, t)$. In accordance with (5.1), $\operatorname{Re}\left(z_{1}\right)$ stays fixed since the disturbance drift along the $X$-axis has been absorbed into the definition of $Z$. About the ultimate fate of $B=\operatorname{Im}\left(z_{1}\right)$, several cases have to be distinguished, depending on the values of $\mu,|k|, S$ and the initial value of $B$ (or $I$ ).

Undoubtedly $B(\infty)$ must be finite, since coth $(|k| B)$ is bounded when $B \geqslant \eta>0$ : the RHS of (7.2) is then bounded by a function independent of $B$, which is itself bounded at $t=\infty$ once integrated. However, $B(\infty)$ may also be zero, as it is to be seen in a moment. To examine the transition between the two cases, we consider situations in which $B(t)$ tends to a small value (possibly zero) as $t \rightarrow \infty$. In the long time limit, th $(|k| B) \cong|k| B$ and (7.2) is solved to give :

$$
t-t_{0}=\int^{B \mathrm{e}^{S t}} \frac{u \mathrm{~d} u}{S u^{2}-u+\mu}
$$

where $t_{0}$ is a constant and $t$ is large by assumption.

If $4 S \mu>1$, the RHS of (7.5) diverges if and only if $B \mathrm{e}^{S t} \rightarrow \infty$ (even though $B|k| \ll 1)$ since (5.4) has no real root in this case. Upon evaluation of the divergent integral in (7.5) one obtains :

$$
S\left(t-t_{0}\right)=\log \left(B \mathrm{e}^{S t}\right)+O(1) .
$$

Therefore $B(\infty)$ is not allowed to vanish if $4 S \mu>1$ and the peak-to-peak amplitude $\Gamma$ saturates at a finite value while the periodic pattern is stretched and its wavelength grows exponentially rapidly as time increases.

If $4 S \mu<1$ however, (5.4) has the two real positive roots $b_{ \pm}$given by (5.5). Then, either (7.6) holds if the initial value of $B$ is large enough $\left(B \mathrm{e}^{S t}>b_{+}\right)$or $B \mathrm{e}^{S t}$ tends to the stable root $b_{-}$. In the latter case, $B(t \rightarrow \infty)=O\left(\mathrm{e}^{-S t}\right)$ and $\Gamma(t \rightarrow \infty)=O(S t)$. One may note that $\Gamma$ increases as the logarithm of the wavelength $2 \pi \mathrm{e}^{S t} /|k|$; this could have been guessed beforehand, since $B \rightarrow 0$ implies that each pole mainly interacts with its complex conjugate, and the disturbance $\phi(X, t)$ corresponding to a two-pole dynamics diverges logarithmically with $X$ (see (5.3)).

On the other hand, whatever $4 \mu S, B(\infty)$ may also be non-zero if $B(0)$ is large enough. Indeed, for a large value of $|k| B$, coth $|k| B \cong 1$, and the following value of $B(\infty)$ obtains :

$$
|k| B(\infty)=|k| B(0)+\frac{|k|}{S}\left(\frac{|k| \mu}{2}-1\right) \text {. }
$$

Since $B(0)=0$ obviously gives $B(\infty)=0$ if $4 S \mu<1\left(B \mathrm{e}^{S t}\right.$ is initially less than $\left.b_{+}\right)$, one concludes that

- if $4 \mu S>1$, then $B(\infty)>0$ for $B(0)>0$

- if $4 \mu S<1$, there exists a critical value $B_{\mathrm{c}}(|k|, S, \mu)$, and the corresponding amplitude $\Gamma_{\mathrm{c}}(|k|, S, \mu)$, such that $B(0)<B_{\mathrm{c}}\left(\right.$ or $\left.\Gamma>\Gamma_{\mathrm{c}}\right)$ implies $B(\infty)=0$ and a diverging peak-topeak amplitude of the wrinkles. Otherwise, for $4 \mu S>1$ or $B(0)>B_{\mathrm{c}}, \Gamma$ always is finite. $B_{\mathrm{c}}$ is easily computed via a numerical integration of (7.2). Typical graphs of $B(\infty)$ as a 
function of $B(0)$ are plotted in figures 3a, b. Given the relation (7.4) $B_{\mathrm{c}}$ also gives the critical amplitude $\Gamma_{\mathrm{c}}$. As far as a two-pole-per-cell dynamics is concerned, these are the non-linear analogs of (3.4) and (3.6).
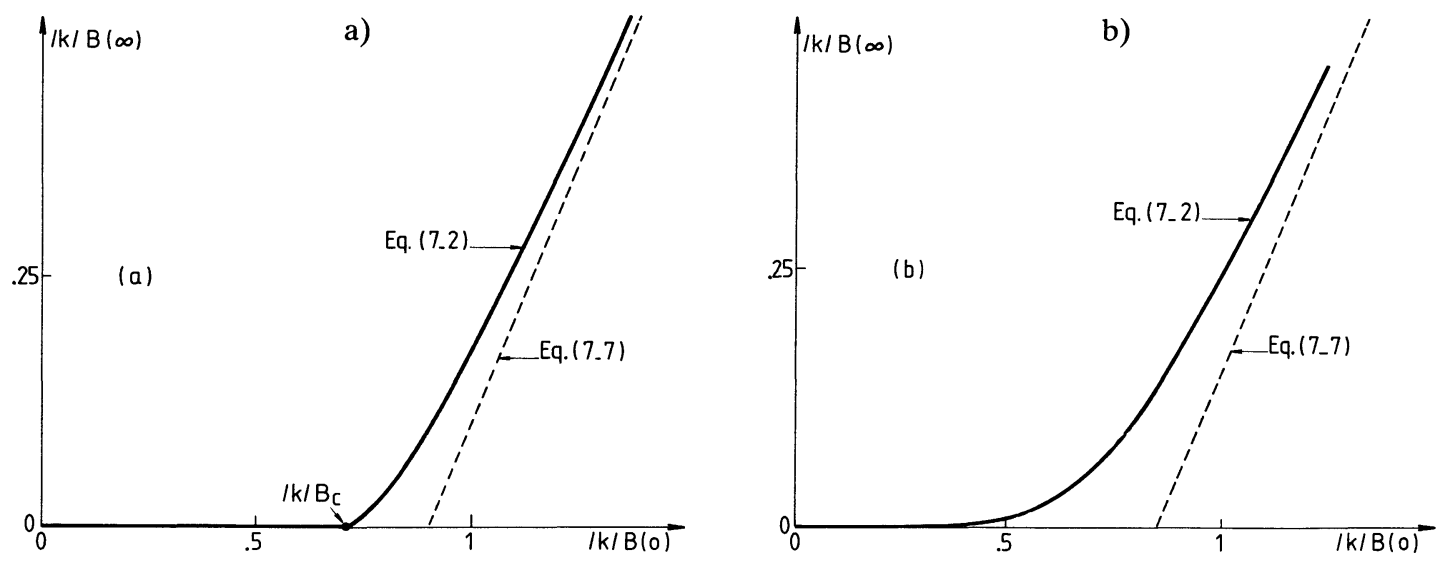

Fig. 3. - Final value of $|k| B$, as given by (7.2), in terms of $|k| B(0)$; figure $3 \mathrm{a}:|k| \mu=$ $0.1, S \mu=0.1$; figure $3 \mathrm{~b}:|k| \mu=0.1, S \mu=0.3$.

For very small values of $S,|k| B_{\mathrm{c}}$ has to be large and can be estimated from (7.7) by requiring that $B(\infty)$ has to be «not-so-large", e.g. $O(1)$, when $B(0)=B_{\mathrm{c}}+O(1)$. This gives :

$$
|k| B_{\mathrm{c}}=\frac{|k|}{S}\left(1-\frac{\mu|k|}{2}\right)+O(1)
$$

and, by (7.4) :

$$
\Gamma_{\mathrm{c}}=O\left(\exp \left(\frac{|k|}{S}\left(\frac{|k| \mu}{2}-1\right)\right)\right)
$$

This is of course the result of the linear analysis, since $|k| B \gg 1$ implies $\Gamma(t) \ll 1$ and a nearly-sinusoidal disturbance. The similarity between (7.2) and (3.2) is best displayed by combining (7.2) and (7.4) to yield :

$$
\dot{\Gamma}=q(t) 2 \operatorname{sh}(\Gamma / 2)\left(1-\mu q(t) \mathrm{e}^{-\Gamma / 2} \cosh (\Gamma / 2)\right)
$$

where $q(t)$ still stands for $|k| \mathrm{e}^{-S t}$; clearly, (7.10) parallels (2.3) and (3.2).

\section{On tip-splitting : stretch vs condensation.}

The front shape disturbance corresponding to (5.3) and to $4 \mu S<1$ may stay about $X=0$ when growing. What could be interpreted as the tip-splitting of an insufficiently curved flame is a rather improbable event however, since it would require the condition $a(0)=0$ to be met. When many $(2 N)$ poles are involved, one may use (4.3), and a summation over the $\alpha$ 's, to show that :

$$
\sum_{\alpha=1}^{2 N} \operatorname{Re}\left(\dot{x}_{\alpha}\right)=S \sum_{\alpha=1}^{2 N} \operatorname{Re}\left(x_{\alpha}\right) .
$$


Accordingly, for any positive $S$ and generic initial conditions $\left(\sum_{\alpha}\left(\operatorname{Re} x_{\alpha}(0)\right) \neq 0\right)$ at least one pair of poles must escape to $X= \pm \infty$ as time elapses. It is believed that they actually all escape ! As an heuristic argument, we provisionally assume that only one pair of poles moves to infinity, the remaining $2 N-2$ poles staying at finite real distances from $X=0$ for $t \gg 1$. For $t \rightarrow \infty$, and because the poles undergo $1 / Z$-interactions, the remaining $2 N-2$ poles would satisfy equations similar to (4.3) (with $\alpha=1 \ldots 2 N-2$ ), with small $O\left(\mathrm{e}^{-S t}\right)$ corrections added to the RHS's. This could not prevent at least one pair of the remaining $2 N-2$ poles from escaping to infinity if they do not satisfy :

$$
\sum_{\alpha=1}^{2 N-2} \operatorname{Re}\left(x_{\alpha}\right)=0 .
$$

The whole process can start all over again, until ultimately all the poles have escaped to $X= \pm \infty$, except if (8.2) is automatically met once the interaction with the escaping pair(s) of poles is over. We have not been able to rule out the last possibility, but we checked our conjecture against numerical integrations of (4.3). Figures $4 a, b, c$, d show snapshots of the pole population when (4.3) is integrated numerically with $N=6, S \mu=0.1$ and initial conditions $x_{\alpha}(0)$ distributed randomly about the imaginary axis.

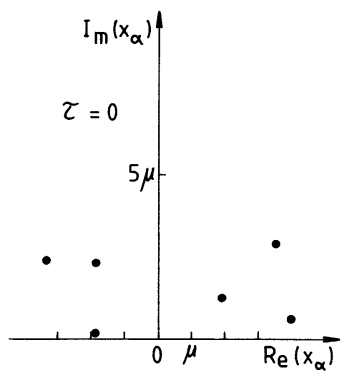

a)

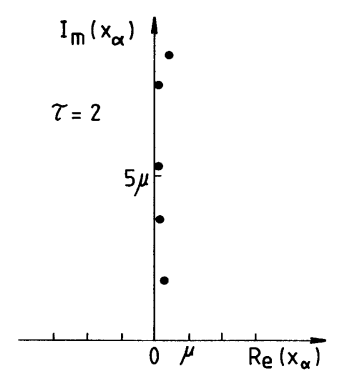

b)

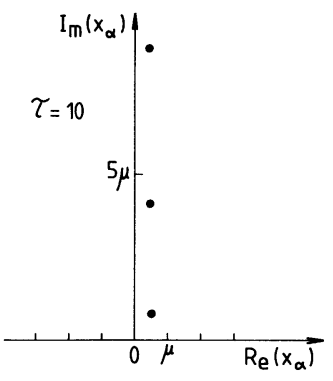

c)

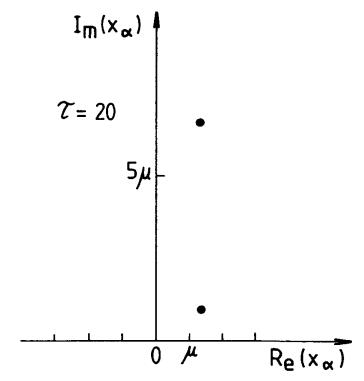

d)

Fig. 4. - Snapshots of the pole evolutions, as given by (4.3), for $N=6, S \mu=0.1$ and different values of $\tau=t / \mu$.

We thus conclude that a symmetric permanent tip splitting is non generic for the class of solutions given by (4.1), no matter how large the number of poles, and whatever the smallness of $S$ ! This does not imply that either the phenomenon of pole condensation along parallels to the imaginary axis $[15,16]$ cannot occur (since solutions of the form $x_{\alpha}=a(0) \mathrm{e}^{S t}+$ $i Y_{\alpha}\left(Y_{\alpha}\right.$ real) are fully compatible with (4.3)) or the steady pole condensation may not exist at $X=0$ for a while. Pole alignments parallel to the imaginary axis may exist after a transient : when two poles $x_{1}, x_{2}$ are close to each other, (4.3) gives $\left(\dot{x}_{1}-\dot{x}_{2}\right)\left(x_{2}-x_{1}\right) \cong 4 \mu$ whatever the value of $S$ and the trend to form vertical alignments [15] is thus preserved. However, the whole pattern(s) must ultimately move to $X= \pm \infty$.

In other words, our result indicates that such condensations at $X=0$ cannot be reached from generic initial conditions on the pole locations.

Once interpreted in the context of the MS equation, it implies that cellular $2 \pi / K$-periodic steady patterns with an extra crest located at $X=\pi / K$ correspond to unstable steady 
solutions to (2.1), in full accordance with the findings of reference [15] obtained by a pseudospectral method. This is also qualitatively compatible with the fact that the steady flames, which propagate in tubes fast enough not to be affected by gravity, do not exhibit split tips permanently, but a single nearly-parabolic tip. Our conclusion, however, disagrees with the results of a simulation quoted in [17], where a two-crest periodic flame pattern was obtained numerically from (2.1) for $\mu K=1 / 10$ and certain initial conditions. It is unclear at the present time whether this discrepancy is due to :

- the choice in [17] of a symmetric initial disturbance, or of an insufficiently long numerical experiment ;

- the class of meromorphic functions $\phi_{X}$ that we consider here, which could be not wide enough to allow for such tip-split solutions.

A somewhat exotic possibility to reconcile the two viewpoints, which has so far not been tested, could be that the lifetime of a « quasi-permanent " split tip increases very rapidly with the number of poles involved at $t=0$. One has also to recall the following fact : using (2.5) as a model to study the stability of steady solutions to (2.1) requires that the latter be seen by the finite-amplitude disturbances as a parabola $S X^{2} / 2$, centered at $X=0$ and with $S$ as curvature irrespective of the disturbance evolutions. In other words, the singularities of the solution to (2.1) are assumed not to move as those corresponding to (2.5) do. Thus, even though the results of this section actually only depend upon $S$ being positive and bounded away from zero and are exact as far as (2.5) is concerned, the above restrictions suggest that they may be applied to the M.S. equation only when the disturbance amplitude is small compared to the peak-to-peak amplitude of the solutions to (2.1).

\section{Concluding remarks.}

The proposed model equation (2.5) seems to be a rather good starting point for studying the non-linear dynamics of curved flame tips and for obtaining non-linear analogs of the conclusions reached by Zel'dovich et al. [4]. Unfortunately we have been able here to display a few of its properties only in a restricted class of exact solutions. Besides this class of poledecomposable solutions, (2.5) undoubtedly also allows for localized disturbances : this is already true for its linearized form, as is shown by adequately superposing elementary solutions of the form (3.1). If true, this conjecture would imply that mild generalizations of (2.5) or (6.1) would describe the dynamics of localized front distorsions about any weaklycurved flame, in a suitably defined lagrangian frame of reference. Whether the fact of being localized changes the way in which the disturbances evolve (as it turned out to be the case for the Mullins-Sekerka instability of an Ivantsov parabola [22]) is still an open problem. In connection with the last point, we further note that mild generalizations of (2.5) exist for which the mode $k=0$ is weakly stable, instead of being merely marginal. The related phenomena of a transient amplification of strong enough disturbances and of a possible balance between disturbance stretching and harmonic generation by non-linearity have been evoked about the flame instability of diffusive origin $(\mu \leqslant 0)$, in the framework of a generalized Kuramoto-Sivashinsky equation [23]; studying them in the context of the Landau-Darrieus hydrodynamical flame instability is a worth investigating, yet unsolved, problem which could be important to understand the dynamics of unstable flames embedded in non-uniform incoming flows.

A last remark is in order. We have so-far considered initial-value problems only ; however, in the context of turbulent flame theory it would also be very interesting to study the flame response to external disturbances. For example, this could be done by adding a random forcing term $u(X, t)$ to the RHS's of (2.1) or (2.5) so as to mimic the influence of small 
velocity fluctuations in the incoming far upstream flow. Such a task has recently been undertaken numerically by Denet [26] about the M.S. equation (2.1), in situations where $u(X, t)$ is a harmonic function of time and space : for several realizations of weak amplitude noises, a quantitative confirmation of the criterion (3.8) was obtained, at least in the cases of not too large cells, for which the numerical noise is indeed negligible and a steady-state, background profile $F(X)$ can be obtained numerically. We also recently proposed a complementary model [27] in which $u(X, t)$ represents a special, spatially inhomogeneous shot-noise that is compatible with a pole-decomposition of $(2.1)$; its influence is merely to implant new complex-conjugate pairs of poles, at random locations $a_{m} \pm i b_{m}$ ( $m=$ $1,2, \ldots)$ and times $t_{m}$, without changing the pole-dynamics between the implants. When a spatially-periodic version of this noise is included in $(2.1), u(X, t)$ reads as :

$$
u(X, t)=\sum_{m} \delta\left(t-t_{m}\right)\left(\psi_{m}(X)-\left\langle\psi_{m}\right\rangle\right)
$$

$\langle$.$\rangle denoting a space-average and the functions \psi_{m}(X)$ being defined according to :

$$
\psi_{m}(X)=-4 \mu \operatorname{Re}\left\{\log \sin \left(\frac{|k|}{2}\left(X-a_{m}-i b_{m}\right)\right)\right\} .
$$

Spatially non-periodic noises are also readily constructed, upon use of $\log \left(X-a_{m}-\right.$ $i b_{m}$ ) functions.

This rapid-distorsion-model is similar in spirit to what was proposed by Chaté [28] and numerically studied in the framework of a discrete model of flame propagation along a 2dimensional lattice; the present suggestion, however, includes the Landau-Darrieus instability as well. The expression (9.1) can also be used as a noise to include in the Burgers' equation (viz. (2.1) without the integral term) which possesses pole-decomposable solutions within the same class as (2.1) does : a comparative study would then give quite interesting information on the role played by the Landau-Darrieus instability in the flame response to a given noise. Similarly, given (4.1) (4.3), one could account for an overall parabolic flame shape, at least in the case of spatially non-periodic noises, upon use of the modified M.S. equation (2.5). Studying whether the same kind of trick can also be extended to spatiallyperiodic noises, via equation (6.1), is the subject of current work, along with the applications of the above studies to unsteady, expanding flames [29].

\section{Acknowledgments.}

The author is greatly indebted to P. Pelcé for fruitful discussions and suggestions, and to P. Cambray for helpful assistance in the numerics.

\section{References}

[1] Uberoi M. S., Flowfield of a flame in a channel, Phys. Fluids 2 (1959) 72-78.

[2] Von Lavante E. and Strehlow A. R., The mechanism of lean limit flame extinction, Combust. Flame 49 (1983) 123.

[3] Pelce-Savornin C., Quinard J. and Searby G., The flowfield of a curved flame propagating freely upwards, Submitted (1987), private communication.

[4] Zel'dovich Ya. B., Istratov A. G., Kidin N. and Librovich V. B., Flame propagation in tubes : hydrodynamics and stability, Combust. Sci. Technol. 24 (1980) 1-13.

[5] LANDAU L. D., On the theory of slow combustion, Acta Physicochim. USSR 19 (1944) 77. 
[6] Darrieus G., Propagation d'un front de flamme ; essai de théorie des vitesses anormales de déflagration par développement spontané de la turbulence, unpublished work presented at La technique moderne, Paris (1938) ; also communicated at the 6th Int. Conf. of Appl. Mech., Paris (1946).

[7] Pelcé P. and Clavin P., Curved front stability, Europhys. Lett. 3 (1987) 907-913.

[8] PelcÉ P., Dynamics of curved fronts (Academic Press) 1988.

[9] SiVASHINSKY G. I., Non-Linear analysis of hydrodynamic instability in laminar flames. Part I : derivation of basic equations, Acta Astronaut. 4 (1977) 1177-1206.

[10] Michelson D. M. and Sivashinsky G. I., Non-Linear analysis of hydrodynamic instability in laminar flames. Part II : numerical experiments, Acta Astronaut. 4 (1977) 1207-1221.

[11] SivAShinsky G. I. and Clavin P., On the non-linear theory of hydrodynamic instability in flames, J. Phys. France 48 (1987) 193-198.

[12] Markstein G. H., Non-Steady flame propagations (Pergamon Press) 1964, p. 198.

[13] Clavin P. and Joulin G., Premixed flames in large scale and high intensity-turbulent flows, $J$. Phys. Lett. France 44 (1983) L1-L12.

[14] Clavin P., Dynamical behavior of premixed flame fronts in laminar and turbulent flows, Prog. Energy Combust. Sci. 11 (1985) 2.

[15] Thual O., Frisch U. and Henon M., Application of pole decomposition to an equation governing the dynamics of wrinkled flame fronts, J. Phys. France 46 (1985) 1485-1494.

[16] LEE Y. C. and CHEN H. H., Non-Linear dynamical models of plasma turbulence, Phys. Scri. T 2 (1982) 41-47.

[17] Michelson D. M. and Sivashinsky G. I., Thermal-expansion-induced cellular flames, Combust. Flame 48 (1983) 211-217.

[18] Bessis D. and Fournier J. D., Pole condensation and the Riemann surface associated with a shock in Burger's equation, J. Phys. Lett. France 45 (1984) L833-L841.

[19] Kruskal M. D., Non-Linear wave motion, Edited by A. Newell (American Mathematical Society, Providence) 1974, p. 61.

[20] Schraiman B. I. and Bensimon D., Singularities in non-local interface dynamics, Phys. Rev. A 70 (1984) 2840-2842.

[21] Gradshteyn I. S. and Ryzhik I. M., Tables of integrals, Series and products (Academic Press) 1980.

[22] Caroli B., Caroli C. and Roulet B., J. Phys. France 48 (1987) 1423.

[23] Sivashinsky G. I., LAw C. K. and Joulin G., On the stability of premixed flames in stagnation point flows, Combust. Sci. Technol. 28 (1982) 153-159.

[24] Cambray P., Deshaies B. and Joulin G., Gaseous premixed flames non-uniform flows, Proc. 70th AGARD Symp., Chania, AGARD preprint $n^{\circ} 422$ (1987), pp. 36.1-36.5.

[25] Joulin G., Existence, stabilité et structuration des flammes prémélangées, Thèse d'état $n^{\circ} 300$, Université de Poitiers (1979).

[26] Denet B., Simulation numérique d'instabilités de fronts de flamme, Thèse de l'Université de Provence (juin 1988).

[27] Joulin G., On a model for the response of unstable premixed flames to turbulence, to appear in Combust. Sci. Technol. (1988).

[28] Chate H., A minimal model of turbulent flame fronts, in Mathematical modeling in combustion and related topics, Eds. Schmidt-Lainé and Brauner, NATO ASI Series $n^{\circ} 140$ (Martinus Nijhoff Pub.) 1988, pp. 441-448.

[29] Joulin G., On the non-linear theory of hydrodynamic instabilities of expanding flames, in preparation. 